\title{
Desempenho de crianças com distúrbio de leitura após o treino auditivo
}

\section{Performance of children with reading difficulties after auditory training}

\author{
Tatiana Yoshie Vatanabe1, Ana Luiza Gomes Pinto Navas², Stefanie Paula Barbosa Mariano³, Cristina Borges \\ Murphy', Alessandra Spada Durante ${ }^{2}$
}

\begin{abstract}
RESUMO
Objetivo: Verificar o desempenho de leitura e da habilidade auditiva de resolução temporal em crianças com dificuldades de leitura, após o treino auditivo. Métodos: Participaram deste estudo 20 crianças na faixa etária de 8 anos, sendo dez escolares com dificuldades de leitura (Grupo Estudo), e dez escolares sem dificuldades escolares (Grupo Controle). Todos os indivíduos foram submetidos à avaliação inicial e reavaliação da resolução temporal auditiva (Padrão de Frequência, Padrão de Duração e Gap in noise (GIN) e de leitura (Protocolo de Leitura Clínica e Teste de Consciência Fonológica - CONFIAS). O Grupo Estudo foi submetido a oito sessões de treinamento auditivo. Resultados: Verificou-se que o Grupo Controle obteve maior desempenho em todos os testes aplicados, quando comparado ao Grupo Estudo, na avaliação inicial e na reavaliação. A diferença do desempenho antes e depois do treinamento auditivo foi significativa para quase todas as tarefas no Grupo Estudo, exceto para a consciência fonológica. Conclusão: $\mathrm{O}$ treino auditivo foi efetivo para a melhora de desempenho nas habilidades auditivas temporais e de leitura em crianças que apresentam dificuldades de leitura.
\end{abstract}

Descritores: Dislexia; Percepção auditiva; Tempo de reação; Criança; Leitura

\begin{abstract}
Purpose: To evaluate reading performance and auditory temporal resolution in children with reading difficulties after auditory training. Methods: Twenty 8 year- old children, 10 with reading difficulties (Study Group) and 10 without reading difficulties (Control Group) were assessed. All subjects participated in an initial assessment and reassessment of auditory temporal resolution (Frequency Pattern, Duration Pattern, and Gap in Noise (GIN) Tests) and reading performance (Clinical Reading Protocol and Phonological Awareness Test - CONFIAS). The Study Group was submitted to eight auditory training sessions. Results: The Control Group showed superior performance on all tests compared to the Study Group at both assessment and reassessment. The difference in performance before and after training was statistically significant for almost all tasks in the Study Group, except for phonological awareness. Conclusion: The auditory training proved effective for improving performance on auditory temporal and reading tasks in children with reading difficulties.
\end{abstract}

Keywords: Dyslexia; Auditory perception; Reaction time; Child; Reading

Trabalho realizado na Faculdade de Ciências Médicas da Santa Casa de São Paulo - FCMSCSP - São Paulo (SP), Brasil, financiado pelo Programa Institucional de Bolsas de Iniciação Científica (PIBIC/CNPq), projeto n 179/09.

(1) Universidade de São Paulo - USP, Faculdade de Medicina, Programa de Pós-Graduação em Neuro-Geriatria em Fonoaudiologia, São Paulo (SP), Brasil.

(2) Faculdade de Ciências Médicas da Santa Casa de São Paulo - FCMSCSP, Curso de Fonoaudiologia, São Paulo (SP), Brasil.

(3) Fonoaudióloga Clínica, São Paulo (SP), Brasil.

(4) Universidade de São Paulo - USP, Programa de Pós-Graduação (Pós Doutorado) em Ciências da Saúde, São Paulo (SP), Brasil.

Conflito de interesses: Não

Contribuição dos autores: $T Y V$ pesquisador principal; $A L G P N$ análise dos dados, correção da redação do artigo; SPBM coleta dos dados; $C B M$ elaboração do protocolo de pesquisa; $A S D$ orientadora.

Endereço para correspondência: Tatiana Yoshie Vatanabe. R. Doutor Cesário Mota Júnior, 61, $8^{\circ}$ andar, Vila Buarque, São Paulo (SP), Brasil, CEP: 01221-020.

E-mail: tvatanabe@yahoo.com.br

Recebido em: 01/07/2013; Aceito em: 28/10/2013 


\section{INTRODUÇÃO}

A habilidade auditiva de resolução temporal é um dos aspectos envolvidos no processamento auditivo temporal ${ }^{(1)}$. A resolução temporal, por sua vez, é definida como a capacidade de detectar intervalos de tempo entre estímulos sonoros, ou detecção do menor tempo que um indivíduo pode discriminar entre dois estímulos sonoros ${ }^{(2)}$.

Embora as relações entre alterações do processamento auditivo, distúrbio de linguagem e distúrbio de aprendizagem sejam complexas, a comorbidade é frequente e, particularmente, muitas crianças com distúrbio de aprendizagem apresentam alteração no processamento temporal ${ }^{(3,4)}$. Essa associação se explica porque alguns indivíduos com alteração do processamento auditivo temporal apresentam déficits na maturação das habilidades auditivas, levando a dificuldades de aprendizagem, leitura e fala ${ }^{(5)}$.

Na literatura, existe evidência de que crianças com alterações nas habilidades auditivas temporais são mais suscetíveis para apresentarem distúrbios de linguagem e aprendizagem, levantando a hipótese que a resolução temporal é fundamental para a percepção de fala ${ }^{(6)}$, assim como para o desenvolvimento adequado de linguagem ${ }^{(7)}$. A correlação entre as alterações nas habilidades auditivas e as habilidades metafonológicas prejudica o uso do mecanismo de análise, síntese, segmentação e manipulação de sons e sílabas, necessários durante a fala e durante a leitura ${ }^{(8,9)}$.

A percepção das informações acústicas para decodificar e codificar os fonemas é fundamental para a aquisição da leitura e, por isso, as crianças com dificuldades em processar os estímulos sonoros da fala, poderão apresentar déficits na segmentação e manipulação da estrutura fonológica da língua, acarretando dificuldades de leitura e escrita ${ }^{(9,10)}$.

Algumas pesquisas sugerem o treino auditivo dessas habilidades alteradas do processamento auditivo temporal, como reabilitação ${ }^{(11,12)}$. O foco principal da utilização do treino auditivo é promover a plasticidade do sistema auditivo central, pois tanto a plasticidade quanto a maturação dependem, em parte, da estimulação. Com a experiência, as vias neurais específicas são ativadas, além de serem facilitadas as estratégias de compensação. A estimulação auditiva por meio do treinamento auditivo, associada a outras intervenções comportamentais, pode aumentar a atividade sináptica, otimizando os circuitos neurais com o aumento do número de neurônios e a mudança no tempo de sincronia neural, favorecendo mudanças comportamentais ${ }^{(13)}$.

Quando o transtorno do processamento auditivo e as dificuldades de aprendizagem são detectados precocemente, há maior possibilidade de garantir orientação adequada aos pais, além de facilitar a conduta de professores no processo de aprendizagem e possibilitar o encaminhamento do aluno à avaliação fonoaudiológica, quando necessário ${ }^{(14,15)}$.

Sendo assim, o objetivo deste estudo foi analisar o desempenho de leitura e da habilidade auditiva de resolução temporal em crianças com dificuldades de leitura, após o treino auditivo.

\section{MÉTODOS}

Esta pesquisa foi aprovada pelo Comitê de Ética (CEP) da Irmandade da Santa Casa de Misericórdia de São Paulo e pela Faculdade de Ciências Médicas da Santa Casa de São Paulo (FCMSCSP), protocolo 179/09. Todos os sujeitos participaram voluntariamente, após os seus responsáveis legais estarem cientes e assinarem o Termo de Consentimento Livre e Esclarecido (TCLE).

Adotamos os seguintes critérios de inclusão neste estudo: residir na grande São Paulo; frequentar uma escola da rede pública e apresentar audiometria tonal e verbal dentro dos padrões de normalidade para a população infantil, segundo estudos anteriores ${ }^{(16)}$. Participaram desta pesquisa 20 sujeitos, 11 do gênero feminino e nove do gênero masculino, estudantes do Ensino Fundamental de uma escola da rede pública da cidade de São Paulo, todos com 8 anos de idade.

Para compor o Grupo Controle, foram selecionadas pelos professores, aleatoriamente, dez crianças sem queixas escolares e, para compor o Grupo Estudo, foram selecionadas, também pelos professores, dez crianças com queixas escolares, incluindo dificuldade para ler.

Os equipamentos utilizados para a avaliação audiológica foram: otoscópio, cabina acústica, audiômetro da marca Madsen ${ }^{\circledR}$, modelo Itera, fone auricular, listas de vocábulos de trissílabos e monossílabos, analisador de orelha média da marca Madsen ${ }^{\circledR}$, modelo Otoflex. Os equipamentos estão de acordo com os seguintes padrões: ANSI S3,6-1989; ANSI S3,43-1992; IEC 645-1 (1992); IEC 645-2 (1993). Para a avaliação dos testes de processamento auditivo, utilizou-se CD player da marca Philips ${ }^{\circledR}$, acoplado ao audiômetro Itera-Madsen.

Foram realizados, na Clínica de Fonoaudiologia da FCMSCSP, para todos os sujeitos, avaliação audiológica básica (Audiometria Tonal, Audiometria Vocal e Imitanciometria), Testes de Processamento Auditivo (Padrão de Frequência Auditec $^{(17)}$, Padrão de Duração - Auditec $\left.{ }^{(17)}\right)$ e Gap in Noise $(\mathrm{GIN})^{(18)}$, Avaliação de Habilidades de Leitura (Protocolo de Leitura e Escrita) ${ }^{(19)}$ e Teste de Consciência Fonológica $(\text { CONFIAS })^{(20)}$.

A pesquisa foi dividida em três etapas: (a) avaliação de ambos os grupos, realizada em duas semanas seguidas; (b) treinamento auditivo (TA), somente com o Grupo Estudo, realizado em oito semanas, uma vez por semana, com sessão de 40 minutos de duração; (c) reavaliação de ambos os grupos, realizada após oito semanas do período do TA, em duas semanas consecutivas.

Para a execução do treinamento auditivo utilizou-se um notebook da marca Accer com o software "Treinamento temporal auditivo com estímulos não verbais e verbais com fala expandida®”, que contém jogos verbais e não verbais, baseados no programa de treinamento auditivo "Fast Forword 
Language"(21). Em cada sessão de treino, foram utilizados 20 minutos para o jogo não verbal ("Jogo do Caco") e 20 minutos para o jogo verbal ("Jogo do Papagaio").

O jogo não verbal ("Jogo do Caco") enfatizou o treino das habilidades de discriminação e ordenação de frequências. Na tela, eram apresentados símbolos que deveriam ser associados ao estímulo sonoro correspondente: $=\mathrm{ou} \neq$, para discriminação dos estímulos; $\uparrow$ ou $\downarrow$, para ordenação, sendo que $\uparrow$ representava o estímulo ascendente, composto por frequências variando de $500 \mathrm{~Hz}$ a $2000 \mathrm{~Hz}$ e $\downarrow$ representava o estímulo descendente, composto por frequências que variavam de $2000 \mathrm{~Hz}$ a $500 \mathrm{~Hz}$ (Figuras 1 e 2). O jogo verbal ("Jogo do Papagaio") foi responsável pelo treino da habilidade de discriminação fonêmica. Os estímulos auditivos eram sílabas apresentando consoante inicial, com tempo de produção expandido e amplificado. Ao ouvir duas sílabas com sons semelhantes, o jogador deveria discriminar e ordenar o par de sons, associando-os à primeira sílaba do desenho correspondente na tela (Figura 3). Ambos os jogos dispunham de adaptação automática de variáveis, como duração do estímulo sonoro, duração do intervalo entre os estímulos e tempo de produção dos fonemas, conforme

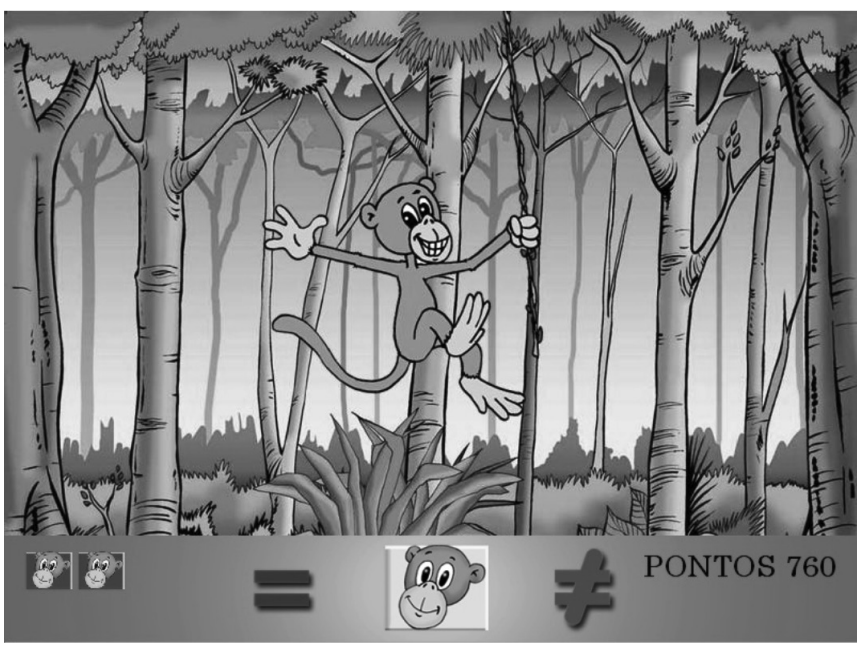

Figura 1. Discriminação de frequência

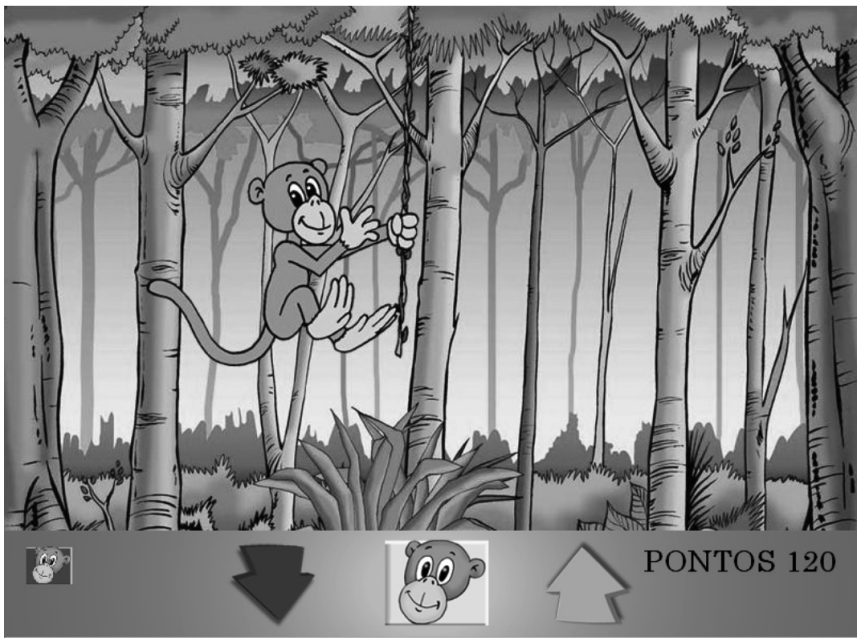

Figura 2. Ordenação de frequência

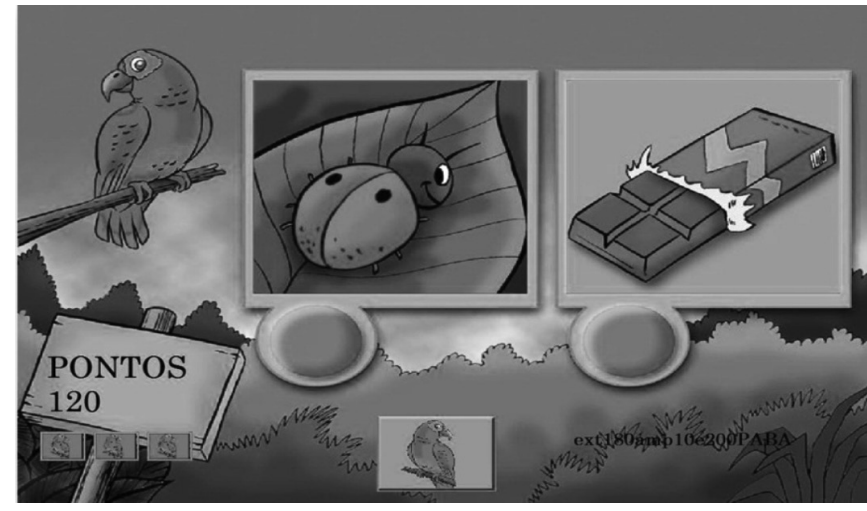

Figura 3. Discriminação e ordenação verbal

desempenho do jogador. Além disso, cada jogador apresentava área própria de acesso ao sistema, para que cada treino fosse personalizado, ou seja, iniciado sempre a partir da última fase atingida pelo jogador na sessão anterior.

Para cada sujeito da pesquisa foram analisadas as seguintes variáveis: grupo (comparação entre Grupo Estudo e Grupo Controle); limiar de detecção de intervalo de silêncio (GIN), por orelha, na avaliação inicial e na reavaliação; porcentagem de acertos nos testes de frequência e de duração, na avaliação inicial e na reavaliação; pontuação na prova de leitura, na avaliação inicial e na reavaliação; pontuação no teste de consciência fonológica (CONFIAS), na avaliação inicial e na reavaliação.

Os dados foram submetidos à análise estatística descritiva e inferencial, com utilização dos seguintes testes estatísticos: teste de Mann-Whitney; Intervalo de Confiança para Média; Análise de Variância com Medidas Repetidas (ANOVA). Em todos os testes foi destacado o nível descritivo, aplicando-se o nível de significância de 0,05 (ou 5\%) para rejeição da hipótese de nulidade.

\section{RESULTADOS}

Houve diferença significativa $(\mathrm{p}<0,05)$ no desempenho do Grupo Estudo, entre a avaliação e a reavaliação, nos testes de Padrão de Frequência e Padrão de Duração (Tabela 1).

Observou-se diferença estatística no desempenho do Grupo Estudo, entre avaliação e reavaliação, no limiar de orelha esquerda ( $\mathrm{p}<0,016$ ) (Tabela 2). Quando comparado o desempenho de ambos os grupos durante a avaliação e a reavaliação, só houve uma diferença estatística no limiar de orelha esquerda $(\mathrm{p}<0,003)$.

Na comparação do desempenho de avaliação e reavaliação do Grupo Estudo e Grupo Controle, no teste de Leitura de Palavras lidas em um minuto e no Tempo Total de Leitura de Palavras, observou-se que apenas o Tempo Total de Leitura apresentou diferença significativa entre os grupos $(p<0,009)$ (Tabela 3).

Com relação ao desempenho de avaliação e reavaliação entre o Grupo Estudo e o Grupo Controle, no CONFIAS, nas tarefas do Nível da Sílaba, Nível do Fonema e Total de Pontos, 
Tabela 1. Desempenho nos testes de Padrão de Frequência e Padrão de Duração por grupo nas fases de avaliação e reavaliação

\begin{tabular}{|c|c|c|c|c|c|c|}
\hline Grupo & Variável & Fase & $\begin{array}{c}\text { Média } \\
\%\end{array}$ & $\begin{array}{c}\text { Mediana } \\
\%\end{array}$ & $\begin{array}{c}\text { Desvio padrão } \\
\%\end{array}$ & Valor de $p$ \\
\hline \multirow{4}{*}{ Grupo Controle } & \multirow{2}{*}{ Padrão de frequência } & Avaliação & 81,5 & 82,5 & 14,0 & \multirow{2}{*}{$0,015^{*}$} \\
\hline & & Reavaliação & 86,5 & 87,5 & 11,1 & \\
\hline & \multirow{2}{*}{ Padrão de duração } & Avaliação & 56,9 & 55,0 & 12,2 & \multirow{2}{*}{$0,035^{*}$} \\
\hline & & Reavaliação & 70,0 & 70,0 & 13,7 & \\
\hline \multirow{4}{*}{ Grupo Estudo } & \multirow{2}{*}{ Padrão de frequência } & Avaliação & 69,9 & 72,5 & 11,9 & \multirow{2}{*}{0,528} \\
\hline & & Reavaliação & 87,0 & 90,0 & 7,9 & \\
\hline & \multirow{2}{*}{ Padrão de duração } & Avaliação & 30,6 & 30,0 & 17,2 & \multirow{2}{*}{$0,005^{*}$} \\
\hline & & Reavaliação & 73,5 & 75,0 & 13,8 & \\
\hline
\end{tabular}

*Valores significativos $(p<0,05)$ - Teste de Wilcoxon

Tabela 2. Desempenho no Teste Gap in Noise (GIN) por grupo e orelha nas fases de avaliação e reavalição

\begin{tabular}{|c|c|c|c|c|c|c|}
\hline Grupo & Orelha & Fase & $\begin{array}{c}\text { Média } \\
(\mathrm{ms})\end{array}$ & $\begin{array}{l}\text { Mediana } \\
\quad(\mathrm{ms})\end{array}$ & $\begin{array}{c}\text { Desvio padrão } \\
\text { (ms) }\end{array}$ & Valor de $p$ \\
\hline \multirow{4}{*}{ Grupo Controle } & \multirow{2}{*}{ Direita } & Avaliação & 5,1 & 5,5 & 1,1 & \multirow{2}{*}{0,851} \\
\hline & & Reavaliação & 5,0 & 5,0 & 0,7 & \\
\hline & \multirow{2}{*}{ Esquerda } & Avaliação & 4,8 & 4,5 & 0,9 & \multirow{2}{*}{0,059} \\
\hline & & Reavaliação & 4,3 & 4,0 & 0,7 & \\
\hline \multirow{4}{*}{ Grupo Estudo } & \multirow{2}{*}{ Direita } & Avaliação & 6,9 & 6,0 & 2,2 & \multirow{2}{*}{0,093} \\
\hline & & Reavaliação & 5,4 & 5,5 & 0,7 & \\
\hline & \multirow{2}{*}{ Esquerda } & Avaliação & 6,8 & 7,0 & 1,9 & \multirow{2}{*}{$0,016^{*}$} \\
\hline & & Reavaliação & 5,1 & 5,0 & 0,6 & \\
\hline
\end{tabular}

*Valores significativos $(p<0,05)$ - Teste de Wilcoxon

Tabela 3. Desempenho nos testes de leitura de palavras e pseudopalavras por grupo nas fases de avaliação e reavaliação

\begin{tabular}{|c|c|c|c|c|c|c|}
\hline Grupo & Variável & Fase & Média & Mediana & Desvio padrão & Valor de $p$ \\
\hline \multirow{4}{*}{ Grupo Controle } & \multirow{2}{*}{$\begin{array}{l}\text { Protocolo de Leitura - } \\
\text { palavras lidas em } 1 \mathrm{~min} \text {. }\end{array}$} & Avaliação & 54,8 & 58,5 & 13,5 & \multirow{2}{*}{0,540} \\
\hline & & Reavaliação & 56,1 & 63,0 & 16,1 & \\
\hline & \multirow{2}{*}{$\begin{array}{l}\text { Protocolo de Leitura- } \\
\text { tempo total }\end{array}$} & Avaliação & $01: 35$ & 01:29 & $00: 33$ & \multirow{2}{*}{0,219} \\
\hline & & Reavaliação & $01: 22$ & 01:09 & $00: 33$ & \\
\hline \multirow{4}{*}{ Grupo Estudo } & \multirow{2}{*}{$\begin{array}{l}\text { Protocolo de Leitura - } \\
\text { palavras lidas em } 1 \mathrm{~min} \text {. }\end{array}$} & Avaliação & 26,5 & 26,5 & 14,3 & \multirow{2}{*}{$0,046^{*}$} \\
\hline & & Reavaliação & 35,0 & 36,0 & 13,9 & \\
\hline & \multirow{2}{*}{$\begin{array}{l}\text { Protocolo de Leitura- } \\
\text { tempo total }\end{array}$} & Avaliação & 04:02 & $02: 43$ & $02: 58$ & \multirow{2}{*}{$0,016^{*}$} \\
\hline & & Reavaliação & 03:06 & $02: 23$ & $01: 45$ & \\
\hline
\end{tabular}

*Valores significativos $(p<0,05)$ - Teste de Wilcoxon

foram observadas diferenças estatísticas $(\mathrm{p}<0,006 ; \mathrm{p}<0,001 \mathrm{e}$ $\mathrm{p}<0,001)$, respectivamente, na comparação da amostra total em todos os itens do CONFIAS (Tabela 4).

\section{DISCUSSÃO}

A partir dos resultados obtidos foi possível verificar que o Grupo Controle teve melhor desempenho nos testes de Padrão de Frequência e Padrão de Duração, quando comparado ao Grupo Estudo. Uma explicação para esses achados seria que, para um bom desempenho nesses testes, a criança precisaria apresentar íntegras as habilidades auditivas de ordenação temporal e transferência inter-hemisférica ${ }^{(22)}$.

Algumas pesquisas apontam, por meio de dados qualitativos do momento da aplicação dos testes do processamento auditivo, a dificuldade dos indivíduos com alteração do processamento auditivo para compreender as instruções de execução de cada teste, típica da própria dificuldade do DPAC. Sendo assim, duas questões poderiam ser levantadas: não se sabe se os resultados alterados do Grupo Estudo se justificariam pela dificuldade de compreender as instruções dadas, ou execução da habilidade, ou se foi exigido um alto nível atencional em cada tarefa, 
Tabela 4. Desempenho no teste de Consciência Fonológica (CONFIAS) por grupo nas fases de avaliação e reavaliação

\begin{tabular}{|c|c|c|c|c|c|c|}
\hline Grupo & Variável & Fase & Média & Mediana & Desvio padrão & Valor de $p$ \\
\hline \multirow{6}{*}{ Grupo Controle } & \multirow{2}{*}{ CONFIAS Sílaba } & Avaliação & 36,5 & 37,0 & 2,9 & \multirow{2}{*}{0,159} \\
\hline & & Reavaliação & 37,6 & 39,0 & 3,9 & \\
\hline & \multirow{2}{*}{ CONFIAS Fonema } & Avaliação & 21,6 & 22,5 & 4,3 & \multirow{2}{*}{$0,008^{*}$} \\
\hline & & Reavaliação & 26,2 & 26,0 & 1,8 & \\
\hline & \multirow{2}{*}{ CONFIAS Total } & Avaliação & 58,1 & 57,0 & 5,8 & \multirow{2}{*}{$0,022^{*}$} \\
\hline & & Reavaliação & 63,8 & 65,5 & 4,6 & \\
\hline \multirow{6}{*}{ Grupo Estudo } & \multirow{2}{*}{ CONFIAS Sílaba } & Avaliação & 33,4 & 35,5 & 5,5 & \multirow{2}{*}{$0,017^{*}$} \\
\hline & & Reavaliação & 35,8 & 38,0 & 5,4 & \\
\hline & \multirow{2}{*}{ CONFIAS Fonema } & Avaliação & 15,0 & 17,0 & 7,1 & \multirow{2}{*}{$0,007^{\star}$} \\
\hline & & Reavaliação & 21,1 & 22,0 & 4,6 & \\
\hline & \multirow{2}{*}{ CONFIAS Total } & Avaliação & 48,4 & 52,0 & 12,1 & \multirow{2}{*}{$0,007^{*}$} \\
\hline & & Reavaliação & 56,9 & 60,0 & 9,3 & \\
\hline
\end{tabular}

*Valores significativos $(p<0,05)$ - Teste de Wilcoxon

tendo em vista que os estímulos auditivos foram apresentados rapidamente ${ }^{(23)}$.

Em um estudo anterior, cujo objetivo foi compreender as características de aprendizagem auditiva em 86 crianças com desenvolvimento típico, por meio de diversas modalidades de estímulos auditivos e de linguagem ${ }^{(24)}$, foi evidenciado que a melhora pós treino auditivo do Grupo Controle, tanto para os testes de audição, quanto de linguagem, ocorreu devido à presença do efeito teste-reteste, sendo impossível concluir até que ponto a melhora pós-treino do grupo estudo foi decorrente exclusivamente do treino ou se também pelo efeito de repetição do mesmo teste.

Os resultados do teste Gap in Noise (GIN), de ambos os grupos, apontaram que os limiares, tanto na orelha direita quanto na orelha esquerda, foram melhores no Grupo Controle do no Grupo Estudo. No entanto, não foram verificadas diferenças significativas entre as orelhas. Esses resultados confirmam outros estudos $^{(25,26)}$. Nesse teste, é avaliada a habilidade de resolução temporal ${ }^{(26,27)}$ e alguns estudos que utilizaram o teste GIN para avaliar o limiar de detecção de gaps em indivíduos com audição normal, encontraram limiares com valores de 3,98 a $6,07 \mathrm{~ms}^{(28)}$. Esses valores de limiares foram compatíveis com os limiares encontrados no Grupo Controle do presente estudo (6 ms).

Algumas pesquisas realizadas ${ }^{(27-29)}$ tentaram relacionar as alterações de fala e escrita com alterações de resolução temporal. Os resultados encontrados mostraram que indivíduos com déficits fonológicos e com dislexia podem apresentar alteração do processamento auditivo temporal e que precisariam de mais tempo para detectar os intervalos de tempo entre os estímulos acústicos ${ }^{(29)}$. Os achados da atual pesquisa concordam com a literatura, pois o grupo de crianças com dificuldades de leitura apresentou limiares de gaps, em ambas as orelhas, maiores do que os do grupo de crianças sem alterações do processamento auditivo e de leitura.

O baixo desempenho obtido pelo Grupo Estudo, em relação aos testes do processamento auditivo temporal, pode indicar a possível relação entre a leitura e o processamento auditivo temporal, concordando com diversos estudos da literatura ${ }^{(29,30)}$. Alguns autores levantaram a hipótese sobre o pior desempenho de crianças com dislexia nos testes de processamento auditivo temporal, lembrando que outros fatores poderiam interferir no desempenho das habilidades fonológicas, como as habilidades cognitivas, sobretudo a capacidade de atenção, e memória de trabalho ${ }^{(30)}$. Todas essas habilidades são essenciais para qualquer aprendizado, principalmente em situações que envolvam a leitura. Com o treino auditivo, as redes neurais foram estimuladas e, possivelmente, melhoraram as habilidades de atenção e memória e de percepção da fala, aumentando a precisão na leitura.

Outra questão a ser destacada é o fato de a melhora do desempenho do Grupo Estudo, após o treinamento auditivo, também ter sido atribuída ao efeito teste-reteste. A fim de investigar melhor essa possibilidade, seria necessária a condução de novos estudos com maior número de sujeitos. Além disso, a incorporação de avaliação por meio de testes eletrofisiológicos poderia minimizar alguns efeitos presentes em testes comportamentais. Estudos futuros deveriam incluir um grupo de indivíduos com a mesma característica dos demais e que fosse submetido a uma condição de estimulação auditiva passiva, para avaliar melhor a real vantagem da estimulação auditiva ativa, para aperfeiçoar o desempenho em leitura, pós intervenção.

\section{CONCLUSÃO}

O treinamento auditivo foi efetivo para a melhora do desempenho nas habilidades auditivas temporais e de leitura em crianças com dificuldades de aprendizagem.

\section{AGRADECIMENTOS}

Agradecemos ao Programa Institucional de Bolsas de 
Iniciação Científica (PIBIC/CNPq), pelo financiamento deste estudo (projeto $\mathrm{n}^{\circ}$ 179/09).

\section{REFERÊNCIAS}

1. American Speech-Language-Hearing Association (ASHA). Central auditory processing: current status of research and implication for clinical practice. Am J Audiol. 1996;5(2):41-52.

2. Shinn JB. Temporal processing: the basics. Hear J. 2003;56(7):52.

3. Breir JI, Fletcher JM, Foorman BR, Klaas P, Gray LC. Auditory temporal processing in children with specific reading disability with and without attention deficit/hyperactivity disorder. J Speech Lang Hear Res. 2003;46(1):31-42.

4. Neves IF, Schochat E. Maturação do processamento auditivo em crianças com e sem dificuldades escolares. Pró-Fono R Atual Cient. 2005;17(3):311-20.

5. Nicholas JG, Geers AE. Effects of early auditory experience on the spoken language of deaf children at 3 years of age. Ear Hear. 2006;27(3):286-98.

6. Olivares-García MR, Peñaloza-López YR, García-Pedroza F, JesúsPérez S, Uribe-Escamilla R, Jiménez-de la Sancha S. Identificación de la lateralidade auditiva mediante una prueba dicótica nueva com dígitos en español, y de la lateralidad corporal y orientación espacial en niños con dislexia y en controles. Rev Neurol. 2005;41(4):198-205.

7. Idiazábal-Alexta MA, Saperas- Rodrígues M. Procesamiento auditivo em el trastorno específico del lenguaje. Rev Neurol. 2008;46(Supl 1):S915 .

8. Mann V, Foy J. Speech development patterns and phonological awareness in preschool children. Ann of Dyslexia. 2007;57(1):51-74.

9. Samelli AG, Mecca FFDN. Treinamento auditivo para transtorno do processamento auditivo: uma proposta de intervenção terapêutica. Rev CEFAC. 2010;12(2):235-41.

10. Pinheiro FH, Capellini SA. Treinamento auditivo em escolares com distúrbios de aprendizagem. Pró-Fono R Atual Cient. 2010;22(1):49-54. 11. Chermak GD, Musiek FE, editors. Handbook of (Central) auditory processing disorder. San Diego: Plural; 2007. Vol. 2, Comprehensive intervention.

12. Tallal P. Improving language and literacy is a matter of time. Nat Rev Neurosci. 2004;5(9):721-8.

13. Zalcman TE, Schochat E. A eficácia do treinamento auditivo formal em indivíduos com transtorno de processamento auditivo. Rev Soc Bras Fonoaudiol. 2007;12(4):310-4.

14. Northen JL, Downs MP. Hearing in children. 3a. ed. Baltimore: Williams \& Wilkins; 1984. p. 89.
15. Moore DR, Rosenberg JF, Coleman JS. Discrimination training of phonemic contrasts enhances phonological processing in mainstream school children. Brain Lang. 2005;94(1):72-85.

16. Balen SA, Liebel G, Boeno MR, Mottecy CM. Resolução temporal de crianças escolares. Rev CEFAC. 2009;11(supl.1):52-61.

17. Auditec. Evaluation manual of pitch pattern sequence and duration pattern sequence. St. Louis; 1999.

18. Musiek FE, Shinn JB, Jirsa R, Bamiou DE, Baran JA, Zaida E. GIN (Gaps-In-Noise) test performance in subjects with confirmed central auditory nervous system involvement. Ear Hear. 2005;26(6):608-18.

19. Capellini SA, Smythe I. Protocolo de avaliação de habilidades cognitivo-linguística: livro do profissional e do professor. Marília: Fundepe; 2008

20. Moojen S, coordenadora. Confias: Consciência fonológica: instrumento de avaliação sequencial. São Paulo: Casa do Psicólogo; 2007. 21. Merzenich MM, Jenkins WM, Jonhston P, Schreiner C, Miller SL, Tallal P. Temporal processing deficits of language-learning impaired children ameliorated by training. Science. 1996;271(5245):77-81.

22. Halliday LF, Taylor JL, Millward KE, Moore DR. Lack of generation of auditory learning in typically developing children. J Speech Lang Hear Res. 2012;55(1):168-81.

23. Zaidan E, Garcia AP, Tedesco MLF, Baran JA. Desempenho de adultos jovens normais em dois testes de resolução temporal. Pró-Fono R Atual Cient. 2008;20(1):19-24.

24. Garcia VL. Processamento auditivo em crianças com e sem distúrbios de aprendizagem [tese]. São Paulo: Universidade Federal de São Paulo; 2001.

25. Hautus MJ, Setchell GJ, Waldie KE, Kirk IJ. Age-related improvements in auditory temporal resolution in reading-impaired children dyslexia. Dyslexia. 2003;9(1):37-45.

26. Perez AP, Pereira LD. O Teste Gap in Noise em crianças de 11 e 12 anos. Pró-Fono R Atual Cient. 2010;22(1):7-12.

27. Schochat E. Desenvolvimento e maturação do sistema nervoso auditivo central em indivíduos de 7 a 16 anos de idade [tese]. São Paulo: Universidade de São Paulo; 2001

28. Van Ingelghem M, Wieringen A, Wouters J, Vandenbussche E, Onghena $\mathrm{P}$, Ghesquière P. Psychophysical evidence for a general temporal processing deficit in children with dyslexia. Neuroreport. 2001;12(16):3603-6.

29. Share DL, Jorm AF, MacLean R, Matthews R. Temporal processing and reading disabilities. Read Writ. 2002;15(1-2):151-78.

30. Murphy CBF, Schochat E. Correlação entre leitura, consciência fonológica e processamento temporal auditivo. Pró-Fono R Atual Cient. 2009;21(1):13-8. 